\title{
PHOTOPHYSICS AND PHOTOREDOX PROCESSES AT LIQUID-LIQUID INTERFACES
}

\author{
Progress Report
}

April 1, 1990 - September, 1992

Professor S.E. Webber

Department of Chemistry

The University of Texas at Austin

Austin, Texas 78712-1167

September 1992

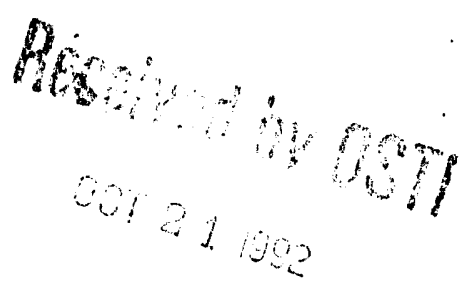

PREPARED FOR THE U.S. DEPARTMENT OF ENERGY

UNDER GRANT NUMBER DE-FG05-86ER13629

\section{DISCLAIMER}

This report was prepared as an account of work sponsored by an agency of the United States Government. Neither the United States Gnvernment nor any agency thereof, nor any of their employees, makes any warranty, express or implied, or assumes any legal liability or responsibility for the accuracy, completeness, or usefulness of any information. apparatus, product, or process disclosed, or represents that its use would not infringe privately owned rights. Reference herein to any specific commercial product, process, or service by trade name, trademark, manufacturer, or otherwise does not necessarily constitute or imply its endorsement, recommendation, or favoring by the United States Government or any agency thereof. The views and opinions of authors expressed herein do not necessarily state or reflect those of the United Stutes Government or any agency thereof. 


\section{Progress Repori on DE-FG05-86ER13629}

\section{"Photophysics and Photoredox Processes at Liquid-liquid Interfaces"}

This report is divided into four sections: 1) Recent work on polymers with covalently bound chromophores, primarily involving triplet state electron transfer quenching; 2) Pyrene and pyrene butyric acid adsorbed onto polystyrene latexes (microspheres); 3) Adsorption and micellization of amphiphilic block polymers with chromophores at the junction between the hydrophobic and hydrophilic part; 4) Adsorption of alternating polymers with grafted "combs" to improve adsorption onto a hydrophobic surface.

\section{1) Photoredox processes in polvelectrolvte-bound chromophores}

This has been the primary thrust of our previous DoE sponsored research. A summary of all of our data and polymer systems appears in Appendix I for the specific case of the zwitterionic viologen 4,4'-bipyridino-1,1' bis(propane sulfonate), abbreviated SPV. Methyl viologen has been used in some of the studies, but the general data is the same and methyl viologen has the disadvantage of forming complexes with aromatic chromophores and/or carboxylate groups.

As has been pointed out in the earlier publications (and the renewal proposal that accompanies this progress report), it has been possible to obtain good charge separation for most singlet state chromophores attached to poly(methacrylic acid) with a long lifetime for the ion-pair, $>1 \mathrm{~ms}$ at low $\mathrm{pH}$. As soon as the polymer begins to deprotonate $(\mathrm{pH}>3.5)$ the yield of charge separation drops to essentially zero, although the quenching efficiency increases dramatically. Pyrene has been an exception case, producing no ion-pairs from the singlet state under any conditions.

For the triplet state very good charge separation has been obtained in all cases and the ion pair also has a long lifetime, in excess of $1 \mathrm{~ms}$ (publications attached). There is no difference in the lifetime of the ion-pair state that results from singlet or triplet state quenching, only in the yield under different $\mathrm{pH}$ conditions. All of this has been rationalized on the basis of spin effects:

$$
2 \mathrm{~S}^{+1} \mathrm{D}^{*}+\mathrm{A} \rightarrow{ }^{2 \mathrm{~S}+1}\left[\mathrm{D}^{+\bullet}, \mathrm{A}^{-\bullet}\right] \rightarrow^{2}\left(\mathrm{D}^{+\bullet}\right)+2\left(\mathrm{~A}^{-\bullet}\right)
$$

The proclivity for the geminate ion pair $\left(2 S+1\left[D^{+\bullet}, A^{-\bullet}\right]\right)$ to recombine into the singlet ground state with the evolution of heat is obviously related to the spin of the parent state and our results imply that spin-mixing in this pair is slower than ion-pair separation and/or charge recombination. The slow recombination of the doublet radicals is a result of their low overall concentration although the polyanion presumably helps repel the ${ }^{2}\left(A^{-\bullet}\right)$ 
species from the region of the cation-radical. We have also noted that aromatic chromophores attached to polyelectrolytes are very easy to photoionize. This creates an experimental difficulty because the excitation laser needs to be strongly attenuated, which reduces the transient absorption signal. If photoionization does occur it can lead to an overestimate of the fundamental yield from reaction (1). In some applications facile photoionization in the aqueous phase could be useful but in the present work it is an undesirable complication.

The fact that the triplet state is an effective precursor to charge separation has been noted by others. ${ }^{1}$ For the triplet state 9-anthracene carboxylate Johansen et al. observed essentially unit efficiency of ion-pair separation ${ }^{1(a)}$. In our work on pyrene butyric acid (discussed in the next section) we find that the triplet state yields ion-pairs with a reasonably long lifetune. We believe that a major factor in the efficient production of ion pairs is the aqueous solvent and dielectric screening of the attraction of the ionic species for each other. In our polymer work the effect of $\mathrm{pH}$ is much less than expected based on the polyanion electrostatic repulsion of SPV- (see Appendix I). For 9-anthracene carboxylate and pyrene butyric acid the $\mathrm{pH}$ must be high in order to achieve significant aqueous solubility. Thus one of the most important advantages of polymer-bound chromophores is to impart aqueous solubility for an otherwise insoluble moiety. Polymers also offer advantages in that they may be cross-linked to for a gel or surface-reacted to form a packing, either of which could be advantageous for practical application.

The attached manuscripts describe the main observations of our more recent polyelectrolyte work. We would like to call attention to several of the specific findings in addition to charge separation. It was noted above that pyrene was an exception in the singlet state work in that no charge separation occurred under conditions where a variety of other similar chromophores displayed high efficiencies. The pyrene moiety had a higher second order fluorescence quenching constant under low $\mathrm{pH}$ conditions than the other chromophores and displayed some "static quenching". This was taken to imply that the pyrene was more exposed to the aqueous phase than the corresponding anthryl derivative. In a joint publication with the PI, M.A.J. Rodgers found that there is no evidence for the formation of $\mathrm{Py}^{+\bullet}$ on the shortest time scale he could measure $(<20 \mathrm{ps})$ (publication attached). We had anticipated that $\mathrm{Py}^{+\bullet}$ would be formed on the ps time scale but that the back-reaction would be much faster than the ca. $10 \mathrm{~ns}$ time scale of the earlier experiments. Evidently there is no Py+• entity formed on any time scale available to us. In the triplet state studies pyrene produced ion pairs with good efficiency, but

\footnotetext{
1 a) Johansen, O.; Mau, A.W-H.; Sasse, W. H. F. Chem. Phys. Lett. 1983, 24, 107; b) Gouterman, M.; Holten, D. Photochem. Photobiol. 1977, 25, 85; c) Olmsted, J.; Meyer, T.J. J. Phys. Chem. 1987, 21, 1649d) Das, P.K., J. Chem. Soc., Faraday Trans. $1,1983,79,1135$.
} 
the kinetics of the SPV ${ }^{\bullet}$ buildup even at low pH show an immediate rise, followed by 2 . slower increase at longer times (see below). This behavior is distinctly different than for anthracene which an immediate buildup of SPV-• absorption at low pH with essentially no further growth (i.e. a "static mechanism") (see Fig. 5 in the attached J. Phys. Chem. manuscript). This is consistent with the idea that the pyrene is in contact with the aqueous phase, even at low $\mathrm{pH}$.
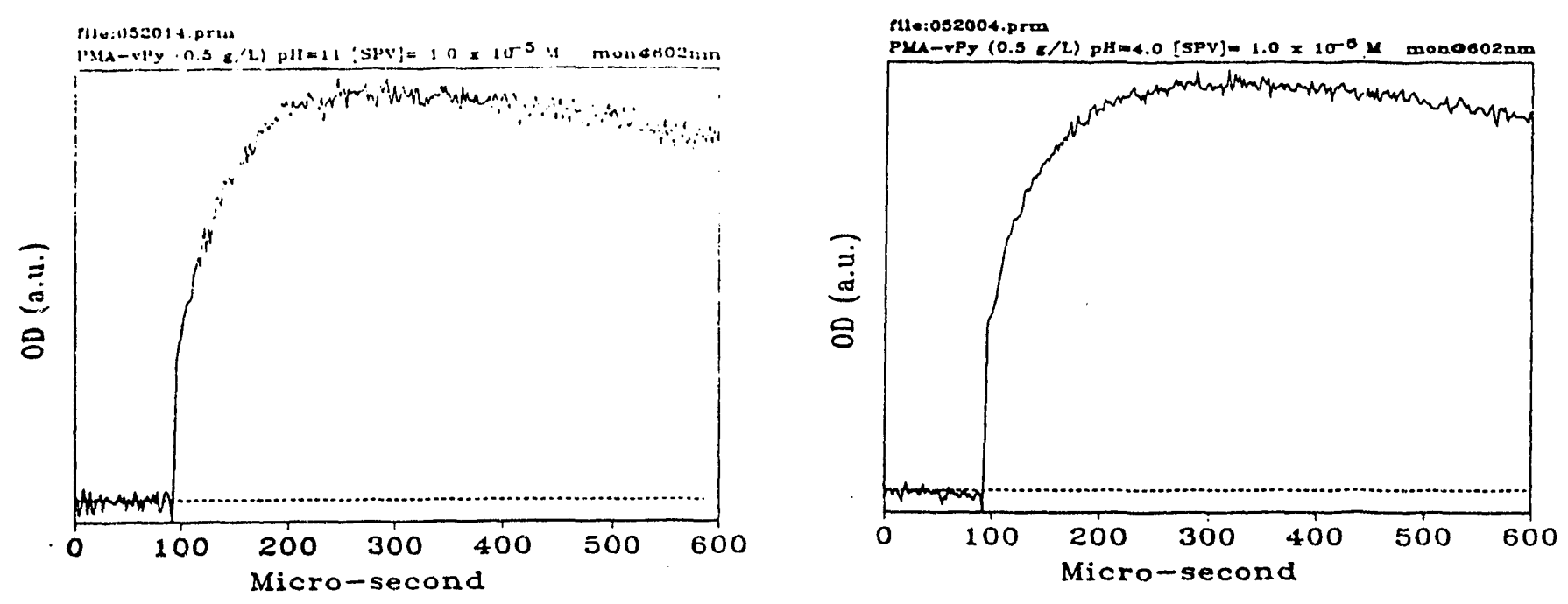

Caption: Optical density at $602 \mathrm{~nm}$ as a function of time for PMA-Py at $\mathrm{pH} 4$ and 11 . Note the similarity of the two curves compared to PMA-Anth (in J. Phys. Chem. article)

The polymethacrylic acid-anthracene case is also interesting. At low $\mathrm{pH}$ where good separation of ion pairs occurred for singlet state quenching there is almost no ion-pair production from the triplet state. The driving force for electron transfer is lower in the triplet state, but the same is true for the triplet state of pyrene. In the triplet state studies the concentration of SPV quencher is much lower (cf. $10^{-5} \mathrm{M}$ with $10^{-2} \mathrm{M}$ ), so it is possible that for the singlet state studies the higher viologen concentration played a "surfactant role" and was associated with the hypercoiled polyacid. These results demonstrate that the chromophore is not an innocent bystander with respect to the polymer conformation at low $\mathrm{pH}$. We have postulated that the collapsed polymethacrylic acid is able to "hydrophobically protect" the chromophore from the aqueous phase. While that idea is consistent with the bulk of the data, it seems evident that the local structure of the protonated polymer around the chromophore may depend on the chromophore. Thus we may state that we do not understand what exactly is involved in "hydrophobic protection". We note that Morishima and co-workers have prepared a variety of modified polymer backbones to explore this concept. ${ }^{2}$ In the proposed new work a structure (3) is suggested that might be interesting in this regard.

2 For a recent review see Morishima, Y. Prog. Pslym. Sci. 1990, 15, 949; 


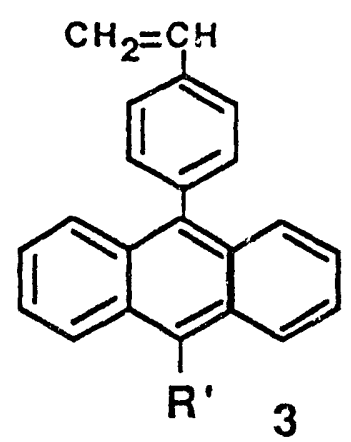

The other observation we made in the J. Phys. Chem. article that may be of general importance is that the triplet state extinction coefficients appear to have been significantly diminished in the aqueous phase. Determination of excited state extinction coefficients is not trivial but we find nearly a factor of two difference. This does not seem to be the effect of simple spectral broadening. We presume it has to do with the effect of solvent polarity in mixing the triplet state with the singlet manifold. Note that if we had used the published organic phase extinction coefficients (which we independently verified) we would have calculated charge separation yields in excess of unity. We suggest that workers should be alert to the same type of solvent effect.

\section{2) Pyrene and pyrene butyric acid adsorbed onto polystyrene latexes (microspheres)}

Polystyrene latexes with surface sulfonate groups can be purchased commercially with very specifie hydrodynamic diameters (Polysciences). While we have made our own "microspheres" using standard emulsion polymerization techniques, we preferred to work with these commerrial samples because they are stated to be surfactant free and do not have any background fluorescence when excited in the pyrene (Py) or pyrene butyric acid (Pyba) region. These microspheres can be loaded with these chromophores by a dialysis technique. Full equilibration takes approximately a week for Py but is much faster for the more water soluble Pyba (at $\mathrm{pH}>10$ ). In both cases the partition coefficient greatly favors sorption $\left(8 \times 10^{4}\right.$ and $1.3 \times 10^{4} \mathrm{~g} / \mathrm{g}$ for Py and Pyba respectively). Py is well-known to change its absorption and fluorescence spectrum in environments of different polarity and in this case Py appears to reside in a non-polar environment, as expected from the microspheres core. Pyba does not have the same fluorescent "fingerprint" as Py, but by comparison of the excitation spectra in different environments it would appear that that the Pyba is also in a non-polar environment. However neither ${ }^{1} \mathrm{Py}^{*}$ nor ${ }^{3} \mathrm{Py} *$ can be quenched by a solution phase species ( $\mathrm{Tl}^{+}$or SPV). The quenching of the Pyba excited states are totally different for Pyba in homogeneous solution (where there seems to be a SPV-Pyba complex formed) and for Pyba adsorbed onto the microspheres (see fluorescence quenching curve below). 


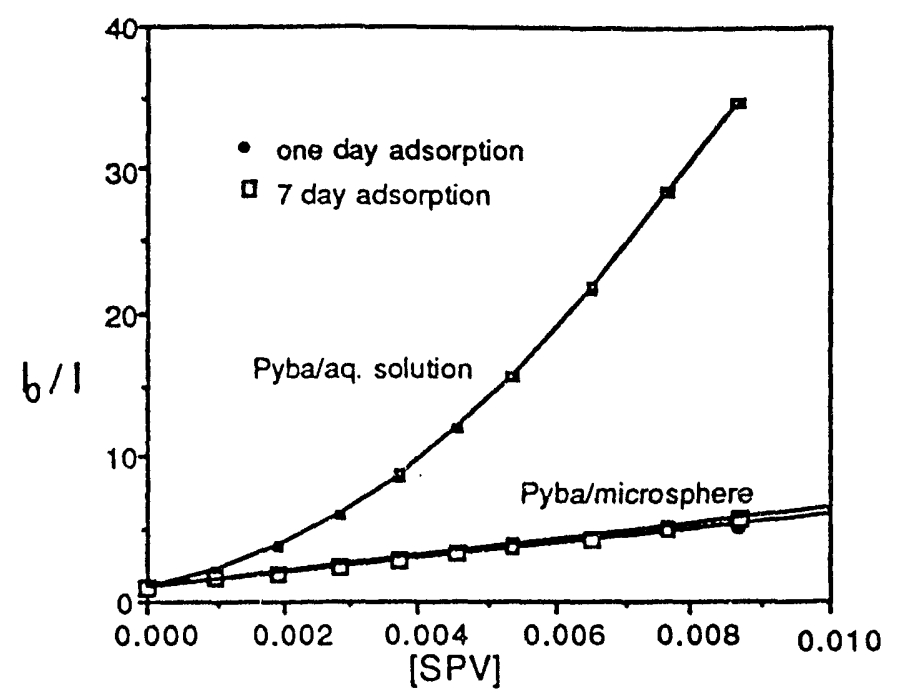

Caption: Comparison of fluorescence quenching of Pyba in homogeneous solution and adsorbed onto microspheres

We had to use $308 \mathrm{~nm}$ ( $\mathrm{XeCl}$ excimer laser) excitation for these electron transfer studies because of the low absorbance of Pyba at $351 \mathrm{~nm}(\mathrm{XeF})$. This limits us to the triplet state because the tail of the absorption spectrum of SPV begins to interfere at the concentrations required for singlet state quenching. The ${ }^{3} \mathrm{Pyba}^{*}$ state was quenched by SPV and the SPV ${ }^{\bullet}$ species was easily observed. The decay of the ${ }^{3} \mathrm{Pyba}^{*}$ state is obvious, as is the growth of the Pyba ${ }^{+\bullet}$, although these is considerable spectral overlap of species in this region, making a quantitative analysis more difficult. We note the ${ }^{3} \mathrm{Pyba}^{*}$ in homogeneous solution also produces $\mathrm{SPV}^{-\bullet}$, although the kinetics are somewhat different (see kinetic curves below) ${ }^{3}$ The rate of loss of the SPV ${ }^{-\bullet}$ is marginally faster in homogeneous solution but both systems are similar to pyrene bound to polymethacrylic acid. Given the quenching behavior and yield of SPV-• we believe that electron transfer is occurring from Pyba moieties that are at the interface of the microspheres. Since the fluorescence properties are consistent with Pyba being in a non-polar environment it seems likely that electron transfer is occurring from the hydrophobic phase to the aqueous phase. We do not know as yet if the $\mathrm{Pyba}^{+\bullet}$ remains associated with the microspheres although the recombination kinetics are different that for homogeneous solution (see below). Thus we take these results as a tentative proof of concept for electron transfer across a polymer-water interface, and this approach will play a central role in proposed future work, albeit with adsorbed polymers rather than small molecules.

\footnotetext{
3 The signal/noise ratio is much better for homogeneous solution tecause of the turbidity of the MS solution.
} 

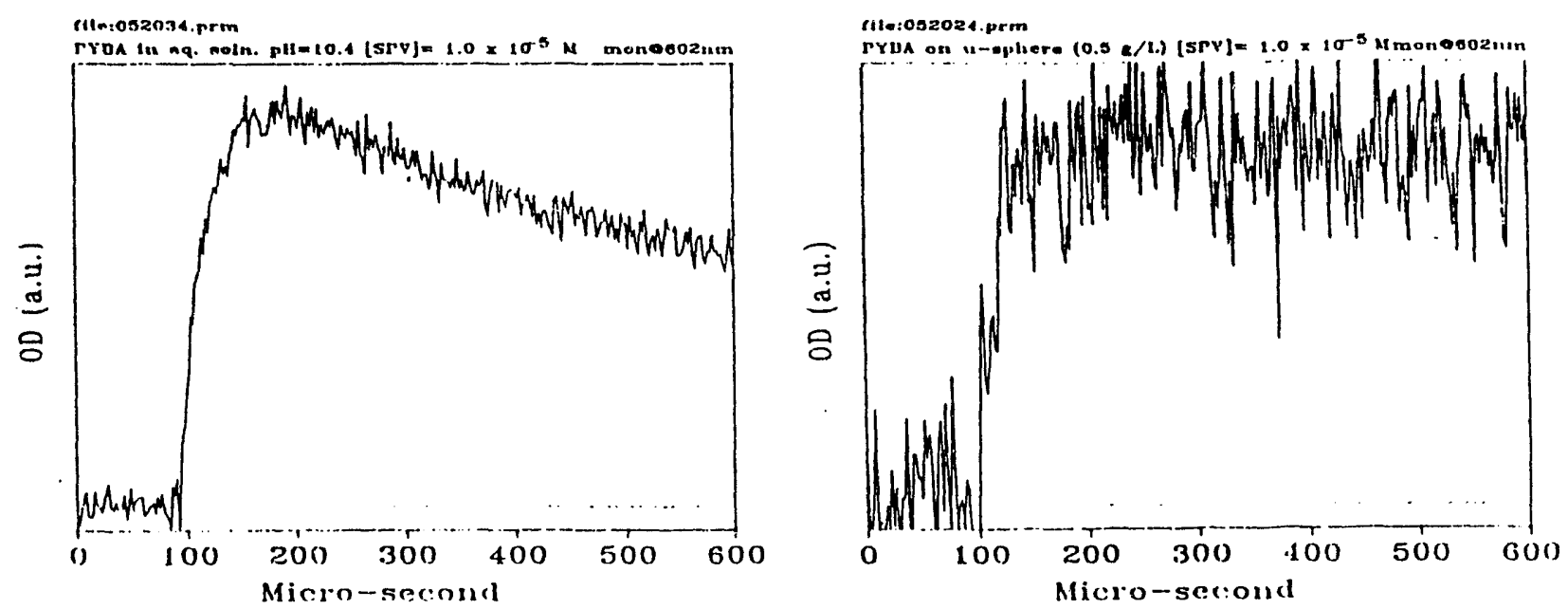

Caption: Absorbance at $602 \mathrm{~nm}\left(\mathrm{SPV}^{-\bullet}\right)$ for Pyba quenched in homogeneous solution or adsorbed onto a microspheres (excitation at $308 \mathrm{~nm}$ ). Note that no reaction occurs for Py absorbed in microspheres

In preliminary work polymethacrylic acid with ca. 1 mole \% of anthracene (PMA-A, the same material used for the singlet and triplet state work in homogeneous solution) was adsorbed onto microspheres under different $\mathrm{pH}$ conditions. The hydrodynamic diameter of the microsphere increased and the quenching kinetics of the anthryl group were strongly modified compared to the homogeneous aqueous phase. Qualitatively these resultsare like the Pyba results described above. This system also yields long-lived ion-pair separation from the triplet state. While the kinetics of $\mathrm{SPV}^{-\bullet}$ formation and decay are slightly modified, the overall behavior is not so different from the polymer in homogenous solution. As in the case of $\mathrm{Pyba}^{+} \cdot$ above, we have not verified that Anth $^{+} \bullet$ is "compartmentalized" in the polystyrene phase.

3) Adsorption of poly(styrene)-blk-poly(2-vinylnaphthalene)-blk-poly(styrene) onto polystyrene films

Polymers of the structure below (abbreviated PS-Naph-PMA) have been synthesized with an average of one naphthalene chromophore placed either at the PS-PMA juncture, as shown below, or at the polystyrene end of the polymer (Naph-PS-PMA). This latter polymer has been shown to produce "null" results.

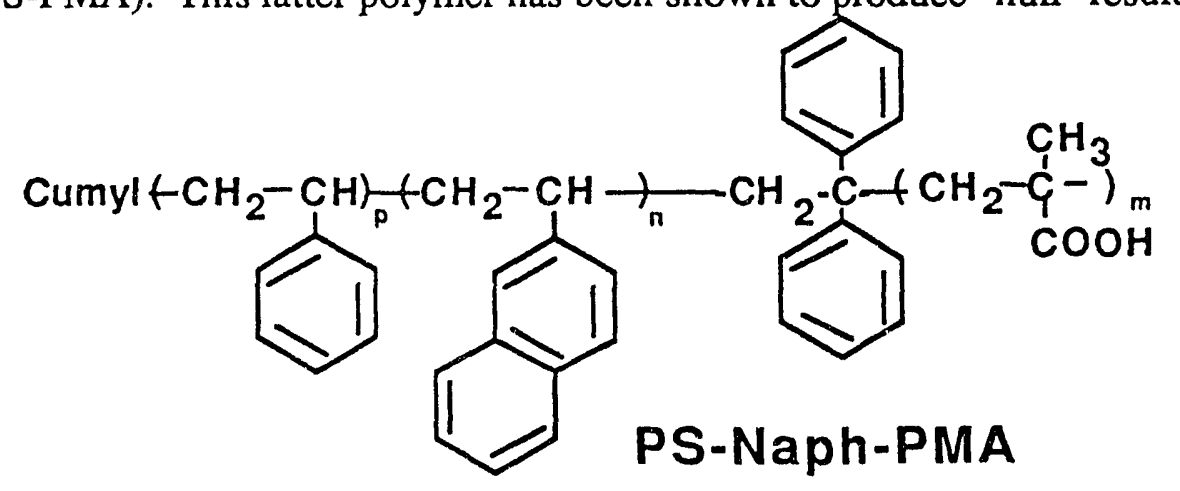


We have studied these polymers extensively with respect to their micelle-forming properties as part of a joint research project with Prof. P. Munk of this Department. ${ }^{4}$ We believe that by replacement of the naphthalene groups with aromatic groups more suitable for electron-transfer processes these micelles should be of interest to our DoE project (this is included in the renewal proposal). In the research so far we have investigated the equilibrium between polymeric micelles, unimers, and surface adsorption, represented below:

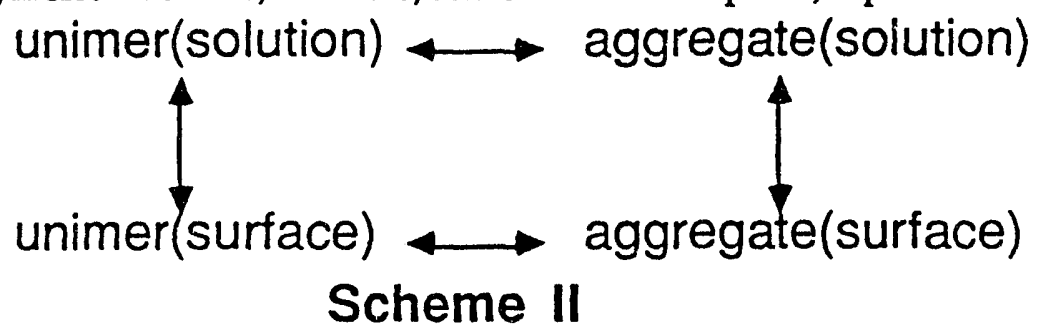

We have found that these micelles are kinetically labile only in $80: 20$ to $60: 40$ dioxane: $\mathrm{H}_{2} \mathrm{O} .5$ For a higher water content these species are kinetically inert. If a suitable dioxane: $\mathrm{H}_{2} \mathrm{O}$ solution of these micelles is placed in contact with a polystyrene film for several hours an apparent irreversible adsorption occurs. The contact angle with water $\left(\Theta_{\mathrm{H}_{2} \mathrm{O}}\right)$ drops from $95^{\circ}$ for untreated polystyrene to ca. $30-40^{\circ}$. This value does not change with extensive water washing. A similar change occurs in Naph-PS-PMA is adsorted onto polystyrene. The. fluorescence spectrum of the naphthalene group is essentially unchanged when in the micelle or on the surface, since it does not display the same environmental sensitivity as pyrene or Pyba, described in the previous section. Because there is approximately one naphthalene per chain there is no significant excimer fluorescence, unlike the case to discussed in the next section.

The naphthalene fluorescence can be quenched by $\mathrm{Tl}^{+}$ions (the viologens, including SPV, would interfere by direct absorption of light). In the case of Naph-PS-PMA no quenching is observed for either the micelles or when adsorbed onto the polystyrene film. We assume that this is because the naphthalene is protected within the hydrophobic core of the micelle or within the polystyrene film. For reasons to be discussed below, we do not believe that intact micelles are adsorbed onto the polystyrene film.

For PS-Naph-PMA micelles the quenching of the naphthalene is particularly facile at high $\mathrm{pH}$ but displays the negative curvature usually associated with the existence of a population of protected chromophores. The

\footnotetext{
4 Our experience to date indicates that mixed micelles can easily be formed with polymers of similar segment length. See for example (a) D. Kiserow, K. Prochazka, C. Ramireddy, Z. Tuzar, P. Munk and S.E. Webber, Macromolecules 25, 461 (1992). (b) K. Prochazka, D. Kiserow, C. Ramireddy, Z. Tuzar, P. Munk and S.E. Webber, Macromolecules 25, 454 (1992).

5 At higher dioxane concentrations these materials can undergo what is known as "anomalous micellization", which is the formation of large intractable aggregates. The physical process that this represents is not understood at present.
} 
quenching is much less efficient at $\mathrm{pH} 7$ and almost nonexistent at $\mathrm{pH}$ 3.5. The quenching of the polystyrene adsorbed naphthalene follows a similar pattern, but with a much smaller degree of quenching at $\mathrm{pH} 10$.

PS-Naph-PMA/PS film

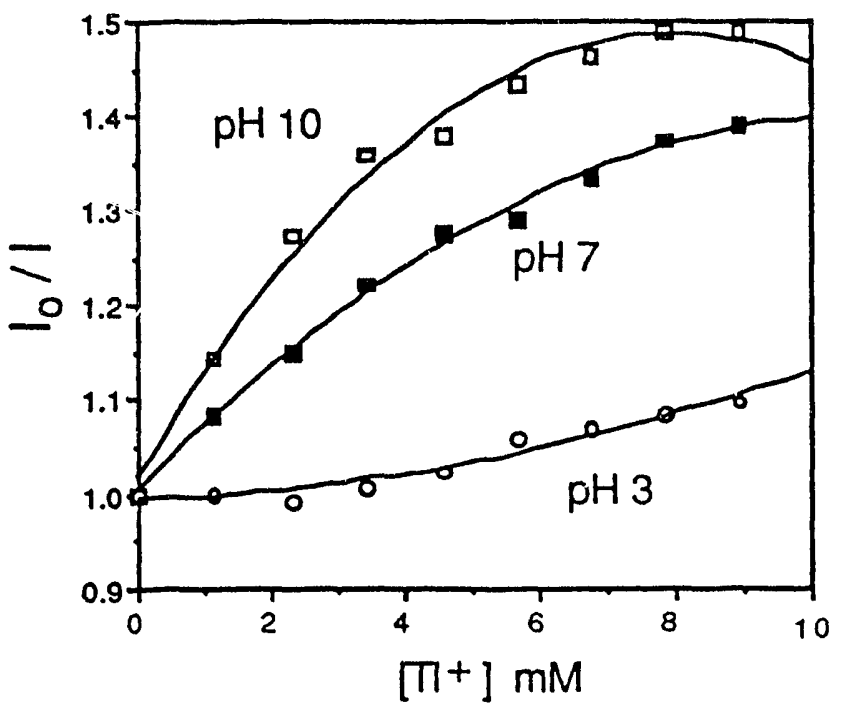

PS-Naph-PMA/micelle

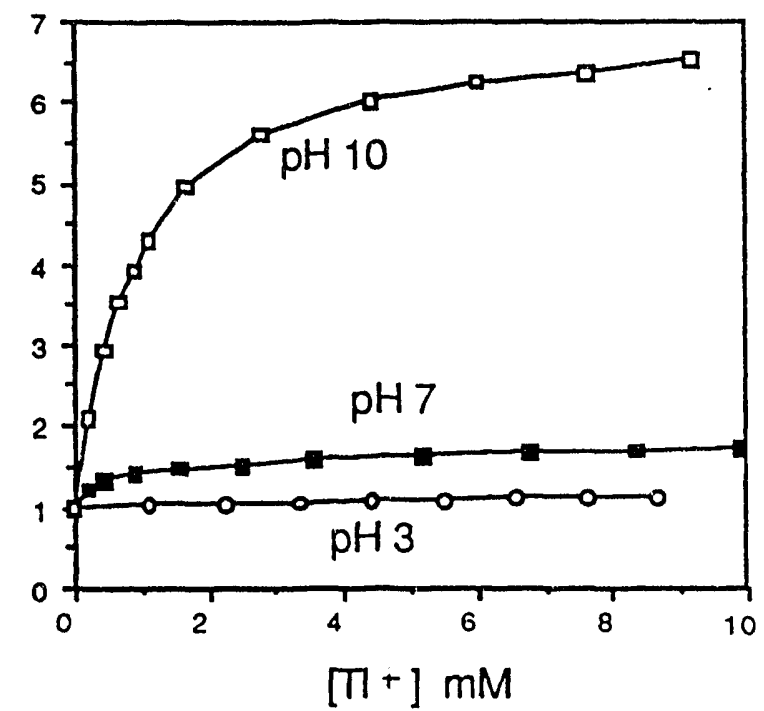

Caption: SV plot of Io/I (ratio of unquenched fluorescence to quenched fluorescence for PS-Naph-PMA/micelle or PS-Naph-PMA/PS film.

Qualitatively it is easy to understand these results. At pH 10 the PMA is completely deprotonated and the polyanion will attract the $\mathrm{Tl}^{+}$species. Furthermore there would be no steric hindrance for approach to the hydrophobic region because the PMA strands will be fully extended. At the lowest pH the PMA coil has collapsed, sterically hindering the approach to the naphthalene and providing no electrostatic incentive for the $\mathrm{Tl}^{+}$ to be near this interface. The only unusual feature in the micelle results is that the difference between $\mathrm{pH} 10$ and 7 is more extreme than is usually found for a chromophore bound at low loadings to PMA. We ascribe this to a "micelle effect" arising from the unusual close packed arrangement of polymers, which creates the potential for a very strong electrostatic field to attract the $\mathrm{Tl}^{+}$to the hydrophobic-hydrophilic interface. The very strong difference in $\mathrm{pH}$ effects between the micelle and the surface adsorbed polymer is why we suggest that it is not an intact micelle that has adsorbed.

When the time dependent fluorescence is examined it is obvious that there is less than $5 \%$ of totally protected naphthalene (i.e. a component that decays with the normal naphthalene lifetime). Thus the negative curvature in the SV plot implies a "hindered approach" to the naphthalene groups. This suggests that the interfacial region is not sharp but instead presents different degrees of exposure to the aqueous phase. We believe the use of molecular photophysics provides a unique insight to these phenomena.

4) The use of alternating polymeis as surfactants and for surface adsorption

Polymers of the following type have been synthesized (denoted P2VN-alt-MA-graft-PS or $\mathrm{C}_{18}$ ) 


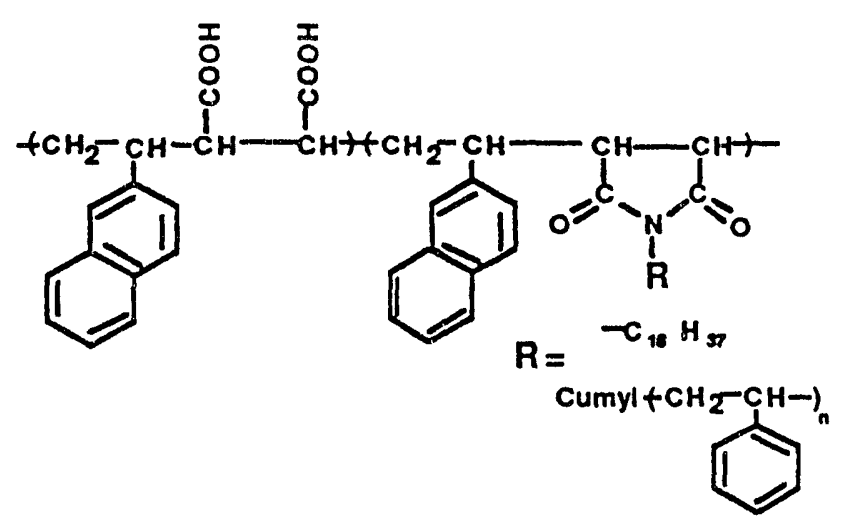

We have also looked at commercial poly(styrene-alt-maleic acid) polymers in which a phenyl replaces the naphthalene group in the above.

A lot of our effort on amphiphilic polymers has involved alternating polymers of this type. We will emphasize the naphthalene-containing polymer because these lend themselves to our normal photophysical experiments. Many of the suriace modification properties of the polystyrene-based polymer are similar.

Initial work on these polymers without the grafts demonstrated that they were very surface active and could be used to make stable octane: $\mathrm{H}_{2} \mathrm{O}$ emulsions with no co-surfactant. The behavior of these polymers in solution was very complex and it was finally realized that one had to be very careful in lowering $\mathrm{pH}$ or changing ionic strength (e.g. dialysis has to be used). Otherwise one can form stable aggregates with hydrodynamic diameters in excess of $1000 \mathrm{~nm}$. The behavior of interfacial surface tension of octane: $\mathrm{H}_{2} \mathrm{O}$ vs. concentration also indicated some aggregation at high concentrations, although not with well-defined structures like the micelles described in the previous sections. These materials would adsorb onto polystyrene or polymethylmethacrylate films in the range of $1-4 \mathrm{mg} / \mathrm{m}^{2}$, depending on the $\mathrm{pH}$, but were easily removed by water washes, unlike the block polymers.

Grafting was accomplished by imidization of either an amino-alkane or a $\mathrm{NH}_{2}$-terminated polystyrene prepared by Dr. C. Ramireddy. ${ }^{6}$ The solution properties of these "comb polymers" are drastically different from the parent polymer and are even more inclined to form aggregates in water or mixed solvents because of their hydrophobic pendent groups. However both can be adsorbed irreversibly onto films. The P2VN-alt-MA-graft$C_{18}$ is easily adsorbed onto polyethylene, reducing $\Theta_{\mathrm{H}_{2} \mathrm{O}}$ from $100^{\circ}$ to $50-60^{\circ}$, and is impossible to remove by washing. Unfortunately we have not been able to carry out photophysical experiments on these materials

\footnotetext{
6 Dr. Ramireddy is a collaborator on the joint micelle project of Prof. Munk and the PI.
} 
because we have not been able to obtain polyethylene that is free of luminescent impurities, even when additive free. ${ }^{7}$

The P2VN-alt-MA-graft-PS does not adsorb directly onto an already-formed polystyrene film. However if a film is formed by the slow evaporation of polystyrene in toluene while in contact with a polymer solution, adsorption does occur that appears to be irreversible. The fluorescence spectrum undergoes a remarkable change, losing almost all excimer component.

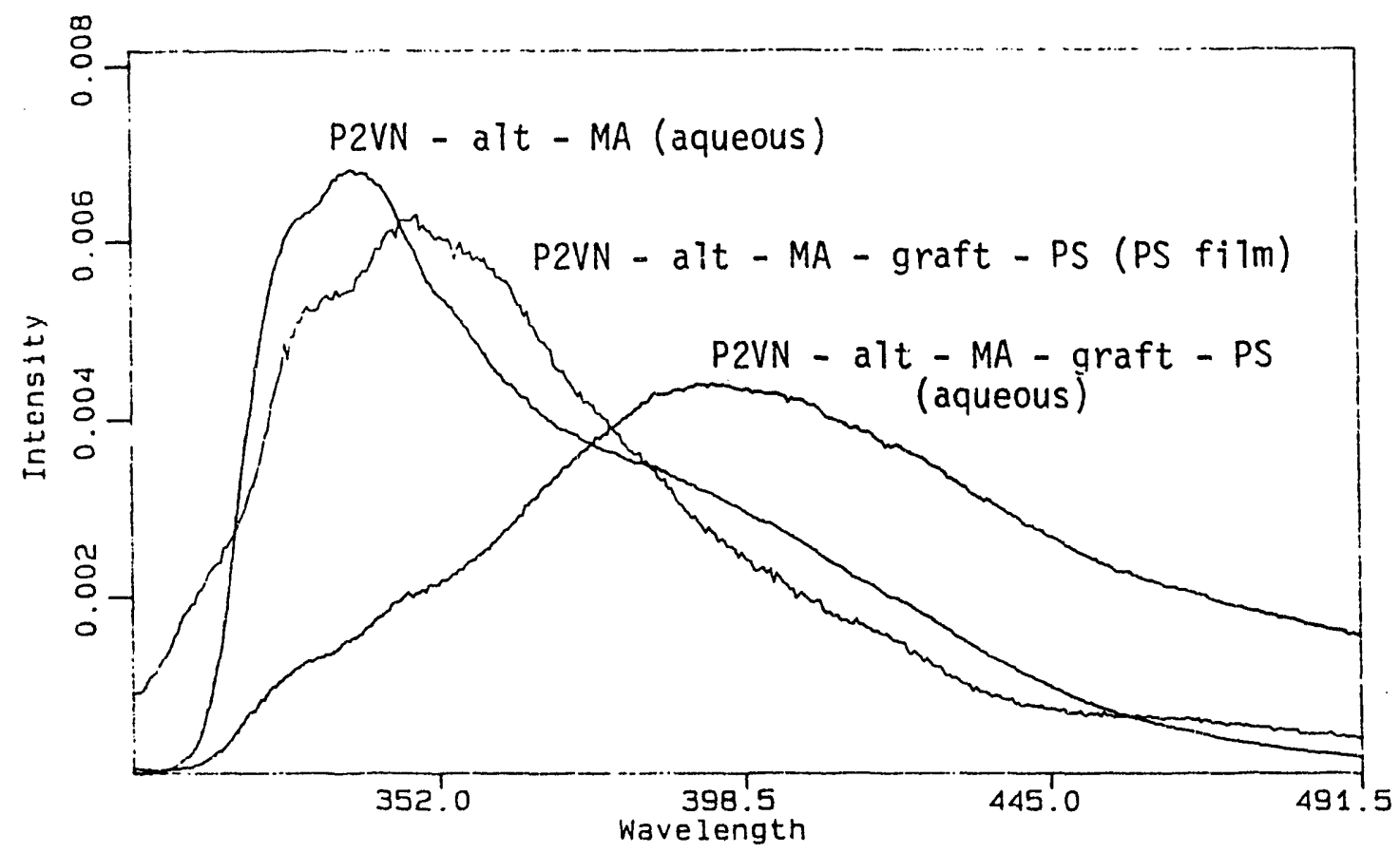

Caption: Comparison of the naphthalene fluorescence for P2VN-alt-MA, P2VN-alt-MA-graft-PS in the solution phase and P2VN-alt-MA-graft-PS adsorbed onto polystyrene film

We take this data to imply that naphthalene are will-isolated from each other. Unfortunately the quality of the film is too poor for reliable $\Theta_{\mathrm{H}_{2} \mathrm{O}}$ measurements, although qualitatively it does not seem that water wets these surfaces as well as the previously mentioned polyethylene case. Quenching measurements imply that ca. 30$50 \%$ of the naphthalene groups are exposed to the aqueous phase.

These polymers can be used as the surfactant in an emulsion polymerization. The results are interesting. We obtain microspheres, similar to those described in part (2) of this Report, with a hydrodynamic diameter of ca. $200 \mathrm{~nm}$ and highly monodisperse (according to light scattering). The stability of these microspheres is sensitive to $\mathrm{pH}$ (at $\mathrm{pH}<4$ they aggregate) implying that the acid groups are important in stabilizing the colloid. The

\footnotetext{
7 Dr. M. Finlayson of DOW Chemical kindly provided polyethylene that is completely free of the usual additives but even this matcrial had significant background fluorescence.
} 
fluorescence of the naphthalene groups is not so different from the solution phase, so the polymer conformation is not the same as when this polymer is adsorbed on polystyrene films. Quenching demonstrates a similar exposure of naphthalene groups the aqueous phase as for the polymer in solution. For the moment we have to assume that polymer aggregates are trapped on the exterior of the microsphere. We think this is a very novel approach to produce photochemically active latexes and one which can be extended to the block polymers as well. This direction is one we propose to follow in new research.

\section{List of manuscripts in preparation}

1. "Interfacial Electron-transfer Quenching of the Triplet State of 1-Pyrene Butyric Acid Adsorbed on WaterSoluble Polystyrene Beads", J-S. Hsiao and S.E. Webber (abstract attached)

2. "Interfacial Electron-transfer Quenching of the Triplet State of Polyelectrolyte-bound Anthracene and Pyrene Adsorbed on Water-Soluble Polystyrene Beads", J-S. Hsiao and S.E. Webber (abstract not available as yet) 3. "Adsorption of Photoactive Amphiphilic Polymers onto Hydrophobic Polymer Films. I. Poly(2vinylnaphthalene-alt-maleic acid)-graft-polystyrene on Polystyrene" T. Cao, W. Yin, and S.E. Webber (abstract attached)

4. "Adsorption of Photoactive Amphiphilic Polymers onto Hydrophobic Polymer Films. II. Poly(styrene) -blkpoly(2-vinylnaphthalene)-blk-poly(methacrylic acid) on Polystyrene" T. Cao, W. Yin, and S.E. Webber (abstract attached)

\section{List of published manuscripts}

1. "Triplet State Electron Transfer in Poly(methacrylic acid) with Covalently BoundPhenanthrene and Naphthalene", P.K. Chatterjee, K. Kamioka, J.D. Batteas, and S.E. Webber, J. Phys. Chem, 95 (2), 960 (1991).

2. "Picosecond Absorption Studies of Photoinduced Charge Separtion in Polyelectrolyte Bound Aromatic Chromophores", M.S. Shand, M.A.J. Rodgers and S.E. Webber, Chem. Phys. Letters 177 (1), 11 (1991). 3. "Triplet State Electron Transfer from Anthracene and Pyrene Covalently Bound to Polyelectrolytes in Aqueous Solution", Jiunn-Shyong Hsiao and S.E. Webber, J. Phys. Chem, 96, 2892 (1992) 
Appendix I. Compilation of results and structures for polyelectrolyte-bound chromophores studies

Table 1. $\phi_{c e}$ data for singlet and triplet redox quenching by SPV

\begin{tabular}{cccc}
\hline Polymer & $\begin{array}{c}\text { Singlet state, } \\
\mathrm{pH} \mathrm{3}\end{array}$ & $\begin{array}{c}\text { Triplet state, } \\
\mathrm{pH} \mathrm{4}\end{array}$ & $\begin{array}{c}\text { Triplet state, } \\
\mathrm{pH} \mathrm{11}\end{array}$ \\
\hline PMA-A & 0.41 & 0.30 & 0.83 \\
PMA-EA & 0.35 & $\mathrm{a}$ & $\mathrm{a}$ \\
PMA-PA & 0.44 & $\mathrm{a}$ & $\mathrm{a}$ \\
PMA-DPA & 0.30 & $\mathrm{a}$ & $\mathrm{a}$ \\
PMA-Per & 0.41 & $\mathrm{a}$ & $\mathrm{a}$ \\
PMA-ANI & 0.18 & NA & NA \\
PMA-Py & 0 & 0.87 & .93 \\
PAA-Py & NA & 0.59 & 0.53 \\
PMS-Py & NA & 0.41 & 0.37 \\
PSS-Py & NA & 0.90 & 0.90 \\
PMA-Naph & NA & $0.59 \mathrm{~b}$ & $1 . \mathrm{C}$ \\
PMA-Phen & NA & $0.45^{\mathrm{b}}$ & 0.46 \\
\hline
\end{tabular}

a. Intersystem crossing yield too low in aqueous solution to carry out the experiment ;

b. $\mathrm{pH}=2$ for these cases

NA = experiment not done

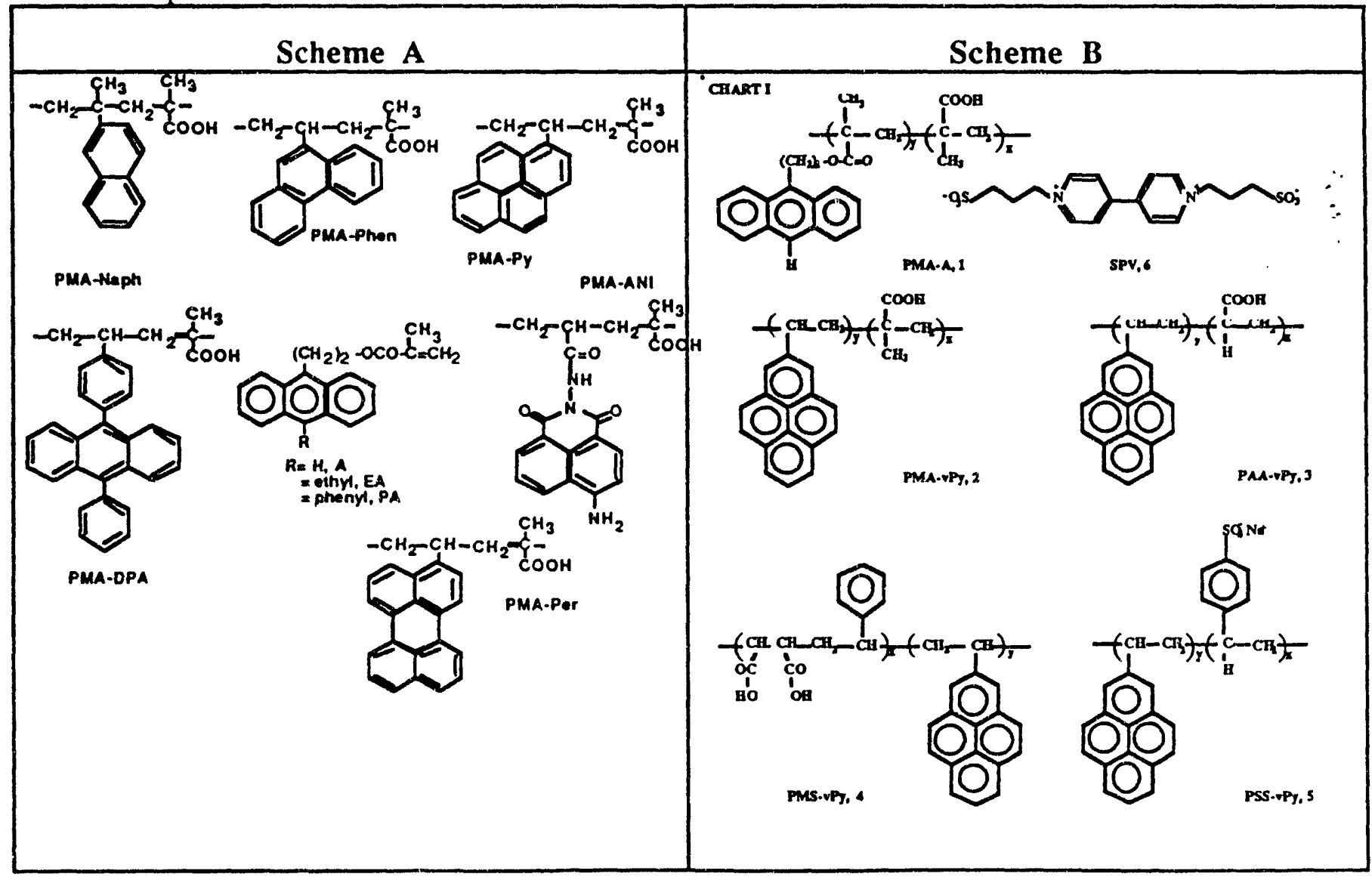



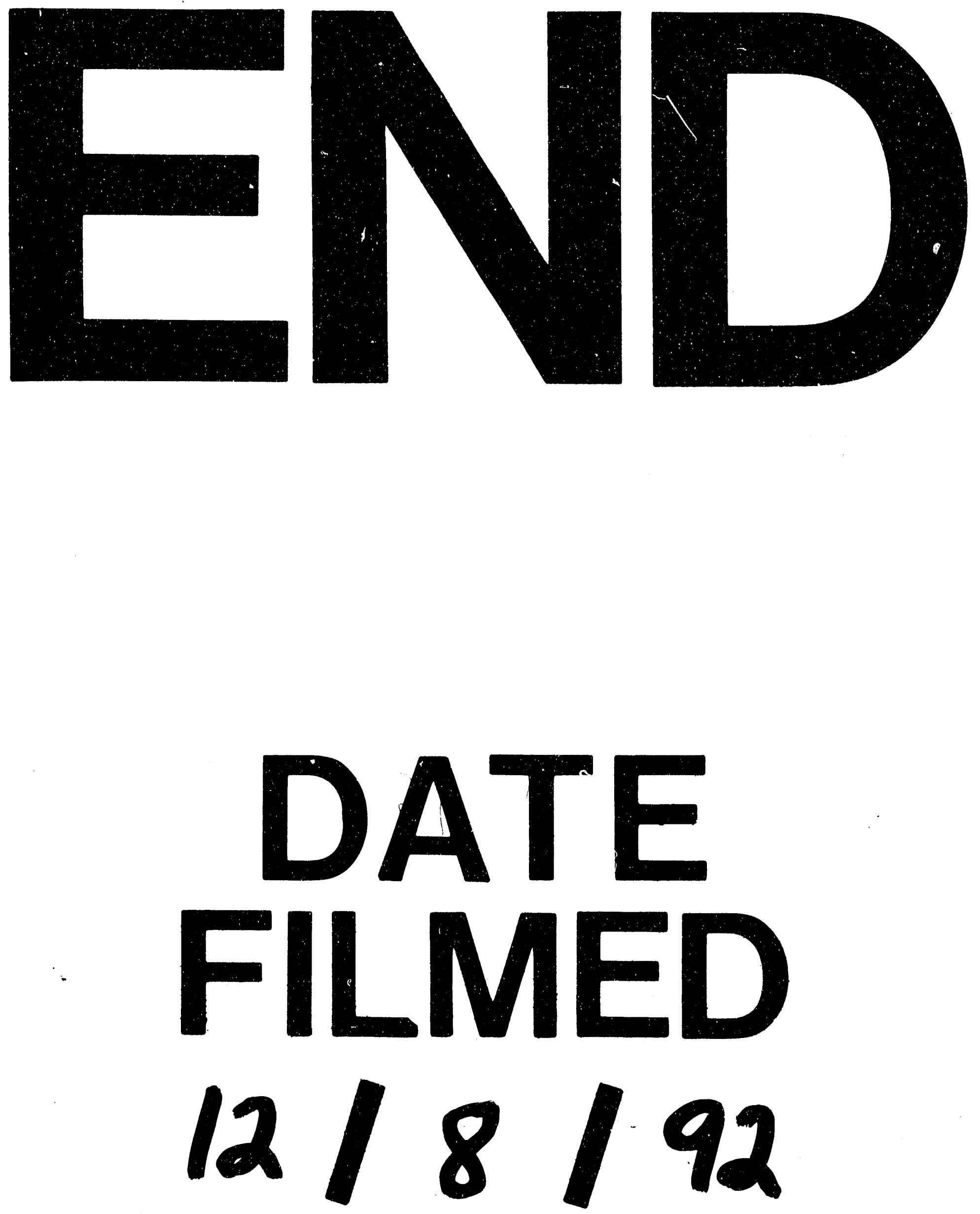
